ISSN $2621-0207$

\title{
Application of a Dielectric Measurement Technique for Calculating Water Loss from Two Texture-contrasting Soils Grown with Upland Rice
}

\author{
Bandi Hermawan ${ }^{1}$, Pajrina ${ }^{2}$, Sumardi ${ }^{2}$, Indra Agustian ${ }^{3}$ \\ ${ }^{1}$ Soil Science Department, University of Bengkulu (Corresponding author) \\ ${ }^{2}$ Agroecotechnology Department, University of Bengkulu \\ ${ }^{3}$ Electrical Engineering Department, University of Bengkulu \\ e-mail:bhermawan@unib.ac.id
}

\begin{abstract}
Most of the water loss from the soil profile occurred through the evapotranspiration process especially when the plant covers were under maximum growth periods. This study aimed to apply a technique of measuring a dielectric variable for calculating soil water content and crop water use in the coarse and medium textured soils grown with upland rice. A couple of wires were inserted into the soil repacked in a 10-kg polybag grown with upland rice, the electrical impedance representing the dielectric value was measured using an instrument called the impedance meter. The impedance values were converted into the soil water content using a nonlinear regression model of $\theta=$ $a . e^{b Z}$ where $a$ and $b$ were constants. Results showed that the proposed technique of measuring the electrical impedance has successfully been applied to calculate the soil water content and the water use by upland rice grown in loamy sand and sandy loam soils. Cumulative water loss from loamy sand was about 4 L plant ${ }^{-1}$ higher for the coarse loamy sand in the first 30 days of a measurement period, but about 10 L plant $^{-1}$ higher for finer sandy loam on the $90^{\text {th }}$ day of the rice growth period. Higher biomass of upland rice in the sandy loam soil could increase the evapotranspiration rates and be the main reason for higher water use in this soil.
\end{abstract}

Keywords: dielectric technique; soil water; texture; upland rice

\section{INTRODUCTION}

In recent years, water resources instead of rainfed approaches have been used for water balance models to evaluate the soil water use by crops and the stabilization of crop production. The models apply soil water content and actual evapotranspiration as two important variables for water balance determination in the agricultural land (Moroizumia et al., 2009). While soil moisture content determines the amount of water remaining in the soil profile, the actual evapotranspiration indicates how much water leaves the profile following the evaporation from the bare soil and the transpiration from the plant. Most of the water loss from the soil profile occurred through the evapotranspiration process especially when the plant covers are under the maximum growth condition. The actual evapotranspiration can be calculated from the microclimate data or water loss from the soil profile when the loss by other processes, such as drainage including percolation and lateral movement of water (Peng et al., 2015), is negligible.
Water loss from the soil profile as a method of determining actual evapotranspiration can be calculated from differences in the temporal soil moisture at a certain period. Soil water content can be determined directly by calculating the weight ratio between water and dry soil, therefore called the gravimetric method (Gardner, 1986). All alternative indirect methods should be calibrated to the standard gravimetric method. Despite its accuracy, the gravi -metric method in soil water content determination has several disadvantages, such as time and labour consuming to provide certain data. Many studies, therefore, have been conducted to develop the alternative indirect methods for soil water determination by measuring the water-related variables including the neutron absorption (Gaze et al., 2002), spectral data measured from reflected radiation (Nagy et al., 2014), and time domain reflectometry (Pan et al., 2012) at the soil profile. In these studies, the behaviour of neutron particles, radiation, and electricity in the ground are used to determine the soil water content. Indirect methods of measuring soil water content have some advantages, such as instantly released data and the in situ 
measurement technique without using laboratory works.

Dielectric characteristics such as the electrical resistivity, capacitance and impedance have increasingly been adopted in engineering, environmental and agricultural studies. Electrical resistivity, for example, is capable of determining the depth of waterfront in the soil profile which is useful to increase the efficiency on the land management practices in terms of cost, time and environmental sustainability (Abidin et al., 2017). Similarly, electrical impedance, which is the measure of the opposition that a circuit presents to the passage of a current when a voltage applied, has strong relations with the amount of water in the soil profile and is capable of calculating plant water use in repacked soils grown with palm oil nursery (Hermawan et al., 2017). The electrical impedance increases when the amount of water in the soil pore space decreases and replaced proportionally by air. However, the degree of the impedance increase per a unit change in soil water becomes more significant when the soil water content is less than 30 percent. Other researchers found that the relations between dielectric and soil water characteristics are influenced by soil types and temperature at the time of measurements (Seyfried and Murdock, 2004).

The amount of water loss from the soil profile by evapotranspiration depends on the proportion of plant cover as indicated by the growth phase and density of plant above the ground surface. Relations between the rate of evapotranspiration and the plant characteristics may be due to the leaf area index as a parameter usually used to predict the photosynthesis process, so that is responsible for the use of soil water by the plant ( $\mathrm{Hu}$ and $\mathrm{Mo}$, 2014). The rate of evapotranspiration increases at higher leaf area index and photosynthesis processes and decreases at the lower index as usually found in the plant water stress regions. The leaf area index is related to the plant biomass, while the biomass production requires a lot of water for the photosynthesis processes. The dependency of plant biomass production on the availability of soil water has been reported elsewhere (Saia et al., 2014). In terms of water use efficiency, the production of plant biomass is expected to uptake more water from the soil, therefore water loss through the plant transpiration should be much greater than that by the evaporation from the soil surface. Increasing water availability to the plant by applying an appropriate irrigation technique may be a good strategy to increase the water uptake by the plant and at the same time to reduce the amount water loss from the soil surface (Šimunek et al., 2016).

Soil texture, classified by the fractions of soil separates including sand, silt and clay has long been known to be an important factor affecting soil water characteristics in relations to the plant water availability and its distribution in the soil profile (Saxton and Rawls, 2006). Coarse and finetextured soils have lower plant water availability compared to medium textured soils. Sandy soils are dominated by larger draining pores but less water-holding smaller pores, while most waters in clay soils are trapped in the micropores and difficult for the plant roots to uptake. Medium textured loamy soils, on the other hand, is dominated by pore spaces that capable of storing water for the plant needs. The proportion of soil separates is of great importance for agricultural and environmental applications such as the landfill and reclamation practices as well as responsible for the presence of water table (Li et al., 2014). Particle size distribution of soil also controls the soil water movement as shown by changes in the wetting pattern size, therefore, is important for the irrigation water efficiency and evapotranspiration needs (Naglic et al., 2014).

This study aimed to apply a technique for measuring the soil dielectric properties, represented by the soil electrical impedance, to calculate the amount of soil water loss by actual evapotranspiration from upland rice grown in two contrasting textured soils. It was assumed that soil water loss by other processes such as vertical and lateral water movement in the soil was neglected in this study. Therefore, temporal changes in soil water content in the planting medium would be responsible for the loss of soil water from the soil surface and plant canopy. The importance of this study is to provide a quick method of measuring soil water content and plant water use in the field by using the easily read data of the electrical impedance.

\section{MATERIAL AND METHOD}

In the current study, the daily actual evapotranspiration from upland rice grown in the polybag was calculated from daily changes in soil water content. Changes in soil water content were determined using a technique of measuring the soil electrical impedance. When subject to water addition by sprinkling, the difference in the soil water content was calculated between the content under the field capacity condition just after the sprinkling and that at the field condition in the next measurement day. Effects of environmental factors such as sunshine hours and wind speed on evapotranspiration as reported by Wang et al. (2014) were negligible in this study since the experiment took place in a tropical region and was conducted in the greenhouse.

The study was conducted in the greenhouse located at the University of Bengkulu, Indonesia (S $03^{\circ} 45^{\prime} 58^{\prime \prime}$; E $102^{\circ} 16^{\prime} 20^{\prime \prime}$ ), from February to 
May 2016. Loamy sand and sandy loam top soils were used as the growing media for upland rice in the polybag. The compositions of sand and silt fractions were 71.29 and $11.48 \%$ for loamy sand and 39.70 and $20.97 \%$ for sandy loam soils, respectively. Ten kilograms of $0-10 \mathrm{~mm}$ aggregates (oven-dry equivalent) were put in the polybag and set in such a way to get the bulk density of about $1.0 \mathrm{~g} . \mathrm{cm}^{-3}$, seeds of upland rice were then sown. A pair of wire was inserted into the soil at the depth of $10 \mathrm{~cm}$, the lowest $5 \mathrm{~cm}$ parts were left uncovered so that enable them to transmit the electrical current through the 5 $-10 \mathrm{~cm}$ soil layers. Therefore the instrument was set to measure the electrical impedance values at the depth of $5-10 \mathrm{~cm}$. The schematic installation of the dielectric-measuring instrument in the experimental polybag was presented in Figure 1.

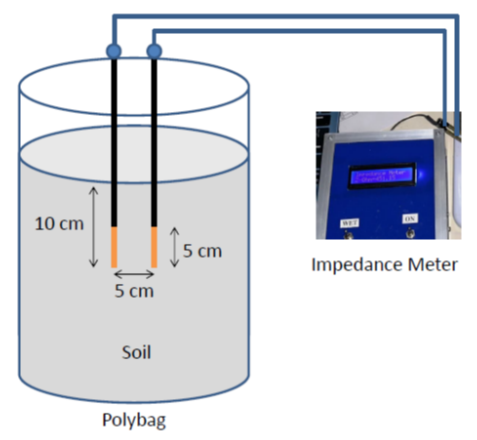

Figure 1. Scheme of instrument installation for the dielectric measurements

The instrument used to measure the electrical impedance was first calibrated by relating the impedance values to measured soil water content. Prior to the seed plantation, the electrical impedance $(Z$, in $\mathrm{k} \Omega)$ ) values were measured and the soilcontaining polybags were weighted to calculate the actual soil water content $\left(\theta\right.$, in g. $\left.\mathrm{g}^{-1}\right)$ in the polybags. The soils were left to evaporate for about 24 hours, $Z$ values were measured and the polybags were reweighted as conducted in the day before. The actual soil water content was calculated as a proportion of the weight of water and oven-dry soil at the time of measurements. Pairs of $Z$ and $\theta$ data were regressed to determine the equation models relating $Z$ to $\theta$. The models were then used to convert the measured $Z$ values to soil water content.

The first $\mathrm{Z}$ measurements were conducted for the water loss calculation one week after the upland rice germination. The soils in the polybags were saturated by adding water to the surface of the soil, the measurements were taken when added water stopped to drain from the bottom of the polybag or when the soil water content in the polybag was in the field capacity condition. The electrical impedance was measured by connecting the upper ends of paired wires to the probes at the impedance-measuring instrument called impedance meter. The instrument generated the direct electrical current at a frequency of $3 \mathrm{~Hz}$, traveling across the $5-\mathrm{cm}$ soil thickness at the depth of $5-10 \mathrm{~cm}$ from the surface. The values of $\mathrm{Z}$ were read from the LCD screen of the impedance meter.

The $Z$ values measured using the proposed instrument were converted to the gravimetric soil water content, showing the weight proportion of water and dry soil in the polybag. When multiplied by the weight of oven-dry equivalent soil $(10 \mathrm{~kg})$, the calculation resulted in the weight of total soil water in the polybag at the time of measurements. The measurements were repeated in the next day, decreases in the weight of water in the soil polybag between two consecutive days of measurements indicated the loss of water by the evapotranspiration for a period of 24 hours. The loss of soil water was then accumulated for 90 days of the measurement period until the crops were in the early stage of generative growth.

\section{RESULT AND DISCUSSION}

The first variable to evaluate in this study was the electrical impedance values of two study soils that were measured daily. Results of the electrical impedance measurements in two-texture contrasting study soils were presented in Figure 2. Temporal variations in the electrical impedance of two study soils were relatively similar in the first 30 days of measurements, the impedance values were less than $5 \mathrm{k} \Omega$ for both soils. However, the differences in the electrical impedance were much higher under the finer sandy loam soil compared to the coarser loamy sand after 30 days of measurements. Since the soil media consisted of solid, liquid and gaseous phases, the magnitude of the impedance values depended on the resistances in the solid phases as well as in the air and water inside the pore spaces. Therefore, variations in the electrical impedance values in the soil media indicated the characteristics of solid, liquid and gaseous phases.

Since the proportions of solid phases in study soils were constant during the temporal measurements, differences in the electrical impedance shown in Figure 2 could be related to the variations in the proportion of air and water occupying the pore spaces. In other words, the temporal changes in the soil electrical impedance during measurement periods were likely related to changes in the water content of study soils. In this case, increasing differences in the electrical impedance between two soils after 
30 days of measurements indicated greater differences in the wetness of two study soils during this period.

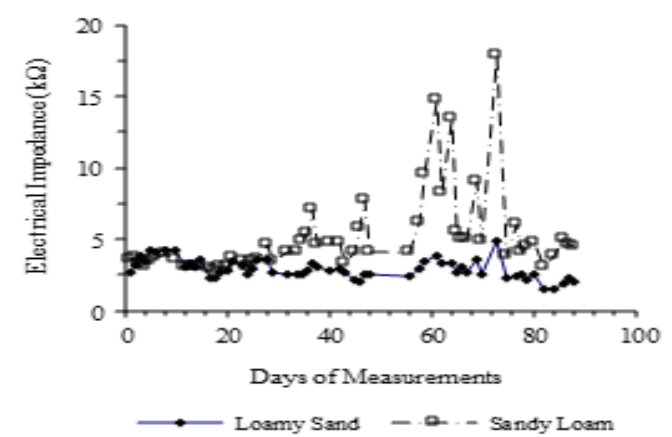

Figure 2. Daily electrical impedance values as measured in the loamy sand $\left(T_{1}\right)$ and sandy loam soils $\left(T_{2}\right)$

Our previous studies on lowland Ultisol and highland Inceptisol found a nonlinear relation between the electrical impedance and water content in the soil (Hermawan et al., 2017). The values of soil water content decreased with increasing values of the electrical impedance, but the slope of the decreased water curve declined at the higher impedance. In the present study, relations between two variables followed the nonlinear exponential equation as follow

$$
\theta=a \cdot \mathrm{e}^{\mathrm{bZ}}
$$

where $\theta$ was soil gravimetric water content (in g.g ${ }^{-}$ ${ }^{1}$ ), $Z$ was the electrical impedance of soil (in $\mathrm{k} \Omega$ ), $a$ and $b$ were constants. The regression analysis on twelve replicates of measurements on two texture-contrasting soils resulted in the equation constants $a$ and $b$ of 0.50 and -0.16 for sandy loam and of 0.55 and -0.06 for loamy soils, respectively. Therefore, Equation (1) was written as

$$
\theta=0.50 . \mathrm{e}^{-0.16 \mathrm{Z}}
$$

for loamy sand and

$$
\theta=0.55 \cdot \mathrm{e}^{-0.06 \mathrm{Z}}
$$

for sandy loam.

The next step of the study was to apply the measured impedance values for the calculation of temporal soil water content as well as the amount of water loss from the soil media. The application of Equations (2) and (3) resulted in the predicted gravimetric water content as a base in calculating the weight of water presenting in the polybags of two study soils. As shown in Table 1, during the three-month growing period of upland rice, the use of water for the sandy loam soil was about 46 percent higher than for the loamy sand soil. Sandy loam soil seemed to become drier much more quickly compared to the loamy sand; therefore, the earlier required more water during the plant growth period. When compared to the electrical impedance data presented in Figure 1, the quick drying processes in the loamy sand was the main reason for highly fluctuating values of the electrical impedance of this soil. The results indicated that the close relations between the electrical impedance and water content of soils as reported earlier (Hermawan et al., 2017) were proved in the current study when the measurement technique for the electrical impedance was used to calculate the plant water use of soils.

Table 1. Daily loss of water $(\mathrm{g})$ in the polybag for two study soils as calculated using Equations (2) and (3)

\begin{tabular}{ccc}
\hline Days & Loamy Sand & Sandy Loam \\
\hline 1 & 0.34 & 0.03 \\
2 & 0.50 & 0.02 \\
3 & 0.42 & 0.11 \\
4 & 0.78 & 0.24 \\
5 & 0.66 & 0.26 \\
6 & 0.75 & 0.33 \\
7 & 0.73 & 0.26 \\
8 & 0.23 & 0.09 \\
9 & 0.32 & 0.09 \\
10 & 0.34 & 0.08 \\
$\ldots$. & $\ldots \ldots$. & $\ldots$. \\
60 & 0.53 & 2.59 \\
61 & 0.23 & 1.21 \\
$\ldots$. & $\ldots$. & $\ldots$. \\
84 & 0.16 & 0.60 \\
85 & 0.21 & 0.46 \\
86 & 0.10 & 0.40 \\
\hline Total & 20.18 & 30.58 \\
\hline
\end{tabular}

The results of this study suggested that changes in the soil electrical impedance following the consecutive 24-hour period of water discharge were more significant when the growth of crops was in the highest growing phase as found in the 60 to 90 days of measurements. Daily changes in the electrical impedance were small in the early stages of crop growth, as shown in the first 30 days of measurements, as well as at the ends of growing periods, probably due to less water consumption by young crops and when the crops were in the generative 
phase, respectively. The results indicated that the electrical impedance had a close relation to the amount of water in the soil media during the growing period of upland rice.

The electrical impedance measurement technique has been successfully applied to calculate the water loss from the two-texture contrasting soils used to grow upland rice. The cumulative water loss from different soil media grown with the upland rice, as calculated using the dielectric measurement technique, was presented in Figure 3. The amount of daily water loss by the evapotranspiration processes were up to $2.5 \mathrm{~L} \mathrm{plant}^{-1}$ as recorded during the maximum growth rates of upland rice in the 60 to 90 days of measurements. During this measurement period, the biomass production of upland rice, represented by crop height, number plants as well as number of panicles per polybag, increased significantly compared to the first month of measurements as shown in Table 2. Results indicated that the amount of water loss by the evapotranspiration process proportionally increased with the biomass production of crops.

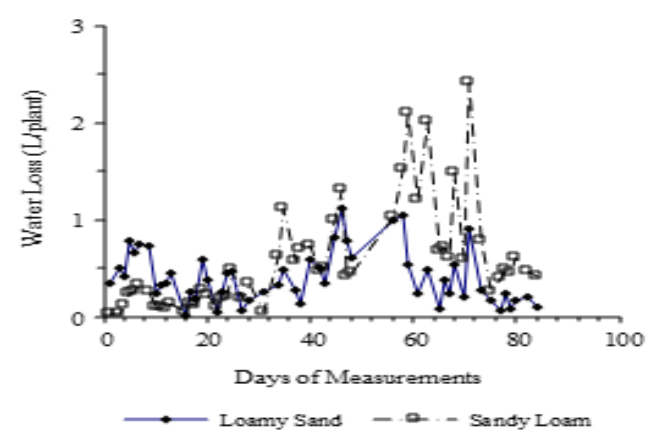

Figure 3. Daily water loss during a growing period of upland rice as measured in loamy sand and sandy loam soils

The final step of the study was to evaluate the ability of the impedance measuring technique to calculate cumulative water loss from the sandy loam and loamy sand soils. The application of the dielectric measurement technique was able to calculate accurately the differences in the water loss between two texture-contrasting soils. As shown in Figure 4, the cumulative soil water loss from the coarse loamy sand was higher than from the medium sandy loam in the first 60 days of measurements. The coarser soil required up to $4.0 \mathrm{~L}$ of the cumulative soil water higher than the finer soil in the first 30 days of measurements. This finding might be related to the fact that less soil surface area for sandy soils could result in soil water repellency, therefore easier to evaporate compared to loamy soils (Nadav et al.,
2013). The trend existed when the soil surface was less covered by the young upland rice in the polybag. However, the differences in the soil water loss decreased with the growing period and the loss from both soils was equal in the $60^{\text {th }}$ day of measurements. After 60 days of measurements, and most of the soil surface has been covered by the plant, the opposite trend was found where the finer sandy loam soil required much more water than the coarser loamy sand soil.

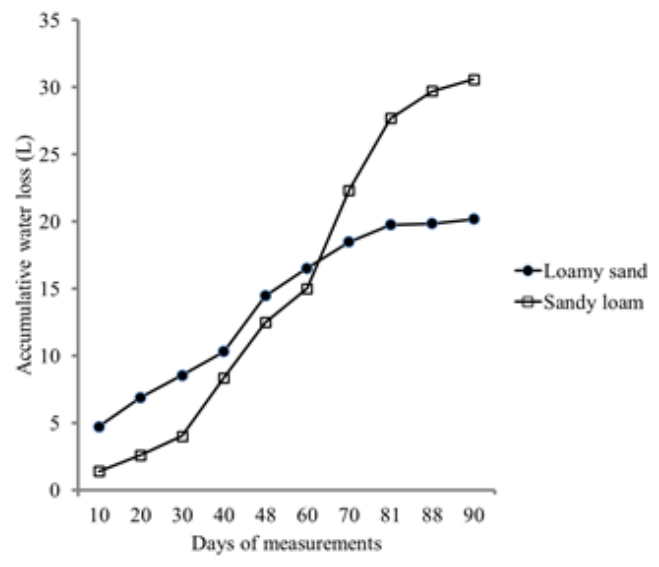

Figure 4. Cumulative water loss during a growing period of upland rice as measured in loamy sand and sandy loam soils

In the first two months of the upland rice growth, the biomass production was low as indicated by the plant height in Table 2. Both soils had a relatively similar height in the second month of measurements, but in the third month of the study the plant height for the sandy loam soil increased significantly to about $10 \mathrm{~cm}$ higher than for the loamy sand soil. A study by Tilly et al. (2015) showed that plant height had a very strong relation to biomass production of rice $\left(\mathrm{R}^{2}: 0.72\right.$ to 0.91$)$ and recommended the variable as a non-destructive estimator for biomass of paddy rice. In particular, the plant height influenced the aboveground biomass significantly as reported by Lou et al. (2016), while this biomass could be responsible for evapotranspiration, therefore the plant height presented in Table 2 might explain the variations in soil water loss between two study soils.

Higher upland rice growth in the sandy loam compared to the loamy sand soil was probably due to differences in the availability of soil water between the two study soils. Dodd and Lauenroth (1997) found that the plant water availability in the $0-30 \mathrm{~cm}$ layers was higher in the sandy clay loam and sandy clay soils than in the loamy sand soil. 
They also proved that when the water availability in the coarse soil was added, the biomass production increased significantly indicating the effects of water availability on the crop biomass production. Soil water availability also influenced the carbon storage as an indicator of biomass production in the nonagricultural land as found by other researchers elsewhere (Álvarez-Dávila et al., 2017). The soil water and the plant biomass production seemed to have two directional relations; the biomass production required the available water in the soil but at the same time promoted the loss of water from the soil.

Table 2. The temporal growth of upland rice in the loamy sand and sandy loam soils

\begin{tabular}{|c|c|c|}
\hline Months & Loamy Sand & Sandy Loam \\
\hline \multicolumn{3}{|c|}{ Crop height $(\mathrm{cm})$} \\
\hline 1 & 77.33 & 69.67 \\
\hline 2 & 90.67 & 92.00 \\
\hline 3 & 110.33 & 122.00 \\
\hline \multicolumn{3}{|c|}{ Number of plants } \\
\hline 1 & 30.67 & 34.00 \\
\hline 2 & 30.00 & 40.00 \\
\hline 3 & 22.33 & 38.33 \\
\hline \multicolumn{3}{|c|}{ Number of panicles } \\
\hline 1 & 2.33 & 0.33 \\
\hline 2 & 20.33 & 28.00 \\
\hline 3 & 22.67 & 33.33 \\
\hline
\end{tabular}

Results of the current study suggested that the application of the proposed electrical impedance technique to calculate the proportion of water in the soil media were beneficial for monitoring the soil wetness quickly and environmentally friendly. While the conventional technique for measuring soil moisture required a lot of works, including the field sampling and the laboratory analysis, the new technique developed in this study offered a simple work to determine the amount of water in the soil. The application of dielectric properties to predict other soil characteristics and deposits has been used by many researchers from various fields of interest. Octava and Yulhendra (2016), for example, have used geoelectric data to determine the deposits of iron ore in the soil (Octova and Yulhendra, 2016), while Paillet et al. (2010) reported good relations were noted between the electrical resistivity with texture, wetness, and cation exchange capacity of the forest soils. Therefore, the application of the proposed dielectric measurement technique could be useful for the calculation of the soil wetness and the soil water loss at various fields of interest.

\section{CONCLUSION}

A technique for measuring the electrical impedance has successfully been applied to calculate the water loss from the loamy sand and sandy loam soils grown with upland rice. The calculation of soil water content $\theta$ from the electrical impedance $Z$ followed the nonlinear regression model of $\theta=$ $a . \mathrm{e}^{\mathrm{bZ}}$ where $a$ and $b$ were constants. The values of $a$ were 0.50 and 0.55 and those of $b$ were -0.16 and 0.06 for loamy sand and sandy loam soils, respectively. Cumulative water loss from loamy sand was about $4 \mathrm{~L}_{\text {plant }}{ }^{-1}$ higher for the coarse loamy sand in the first 30 days of the measurement period, but about $10 \mathrm{~L} \mathrm{plant}^{-1}$ higher for finer sandy loam on the $90^{\text {th }}$ day of the rice growth period. At the end of growing period, higher biomass production of upland rice in the sandy loam soil could increase the evapotranspiration rates and be the main reason for higher water use in this soil.

\section{Acknowledgements}

We thank Syahdano Yamadasi, a student of the Electrical Engineering University of Bengkulu for the instrumentation works. The research was partly funded by the Ministry of Research, Technology and Higher Education, Republic of Indonesia, Contract Nomor: 888/UN30.15/LT/2016, $14^{\text {th }}$ March 2016.

\section{References}

Álvarez-Dávila, E., L. Cayuela, S. González-Caro, A.M. Aldana, and P.R. Stevenson. (2017). Forest biomass density across large climate gradients in northern South America is related to water availability but not with temperature. PLOS ONE 12(3): e0171072. DOI : https:// doi.org/10.1371/ journal.pone.0171072.

Abidin, M.H.Z., A. Madun, S.A.A. Tajudin, M.F.T. Baharuddin, M.F. Yusof, M.N. Zakaria, and S.N. Rahmat. (2017). Evaluation of unknown tube well depth using electrical resistivity method. MATEC Web of Conferences 103, 10 pp (April 2017). DOI: https://doi.org/10.1051/matecconf/ 201710307002.

Dodd, M.B. and W.K. Lauenroth. (1997). The Influence of Soil Texture on the Soil Water Dynamics and Vegetation Structure of a Shortgrass Steppe Ecosystem. Plant Ecology, 133, 1328.

Gardner, W. 1986. In A. Klute (editor). (1986). Methods of Soil Analysis. Part 1: Physical and 
Mineralogical Methods. Second edition. ASA, Inc., SSSA, Inc., Madison, Wisconsin, USA. pp. $493-544$.

Gaze, S.R., M.A. Stalham, and E.J. Allen. (2002). Accuracy of the neutron probe for measuring changes in soil water storage under potatoes. The Journal of Agricultural Science, 138(2), 135-152.

Hermawan, B., E. Suparjo, K.S. Hindarto, R. Silalahi, and F. Barchia. (2017). A Quick Dielectric Method to Determine Insitu Soil Water Content for Precision Water Use under Sustainable Agricultu ral Practice. Int. J. Adv. Sci. Eng. and Info. Tech., 7 (3), 910-915.

Hermawan, B., I. Agustian, H. Suhartoyo, and Sukisno. (2017). A Simple Alternative Instrument for Measuring Temporal Soil Water Content in Repacked Soils. The $2^{\text {nd }}$ Conference on International Symposium on Sustainable Agriculture and Agroindustry (ISSAA 2017), Walailak University, Nakhon Si Thammarat, Thailand $\left(28^{\text {th }}-\right.$ $29^{\text {th }}$ March 2017).

Hu, S. and X. Mo. (2014). Prediction of crop productivity and evapotranspiration with two photosynthetic parameter regionalization methods. Journal of Agricultural Science, 152, 119 $-133$.

Li, X., S.X. Chang, and K. F. Salifu. (2014). Soil texture and layering effects on water and salt dynamics in the presence of a water table: a review. Environmental reviews, 22(1), 41-50.

Lou, Y., Y. Pan, C. Gao, M. Jiang, X. Lu, and Y.J. $\mathrm{Xu}$. (2016). Response of plant height, species richness and aboveground biomass to flooding gradient along vegetation zones in floodplain wetlands, northeast china. PLoS ONE 11(4): e0153972. DOI: https://doi.org/10.1371/journal. pone. 0153972.

Moroizumia, T., H. Hamadab, S. Sukchanc, and M. Ikemotod. (2009). Soil water content and water balance in rainfed fields in Northeast Thailand. Agricultural Water Management, 96: 160 -166 .

Nadav, I., J. Tarchitzky, and Y. Chen. (2013). Induction of soil water repellency following irrigation with treated wastewater: effects of irrigation water quality and soil texture. Irrig Sci., 31: 385-394. DOI https://doi.org/10.1007/s00271 -011-0316-y.

Naglic, B., C. Kechavarzi, F. Coulon, and $\cdot$ M. Pintar. (2014). Modelling of soil water distribution under surface drip irrigated hop. Irrig Sci., 32, 421436.

Nagy, A., P. Riczu, B. Gálya, and J. Tamás. 2014. Spectral estimation of soil water content in visi- ble and near infra-red range. Eurasian Journal of Soil Science, 3(3), 163-171.

Octova, A. and D. Yulhendra. (2016). Iron ore deposits model using geoelectrical resistivity method with dipole-dipole array. MATEC Web of Conferences 101, 6 pages (March 2016). DOI: https://doi.org/10.1051/matecconf/ 201710104017

Paillet, Y., N. Cassagne, and Jean-jacques Brun. (2010). Monitoring forest soil properties with electrical resistivity. Biology and Fertility of Soils; Heidelberg, 46(5), 451-460.

Pan, F., Y. Pachepsky, D. Jacques, A. Guber, and R.L. Hill. (2012). Information and complexity measures applied to observed and simulated soil moisture time series. Soil Sci. Soc. Am. J., 76(3), 829-844.

Peng, S., Y. He, S. Yang, and J. Xu. (2015). Effect of controlled irrigation and drainage on nitrogen leaching losses from paddy fields. Paddy and Water Environment, 13(4), 303-312.

Saia, S., G. Amato, A.S. Frenda, D. Giambalvo, Dario, and P. Ruisi. (2014). Influence of Arbuscular Mycorrhizae on Biomass Production and Nitrogen Fixation of Berseem Clover Plants Subjected to Water Stress. PLoS One; San Francisco, 9(3) (Mar 2014).

Saxton, K.E. and W.J. Rawls. (2006). Soil Water Characteristic Estimates by Texture and Organic Matter for Hydrologic Solutions. Soil Sci. Soc. Am. J., 70(5), 1569-1578.

Seyfried, M. and M.D. Murdock. (2004). Measurement of Soil Water Content with a 50$\mathrm{MHz}$ Soil Dielectric Sensor. Soil Sci. Soc. Am. J., 68(2), 394-403.

Šimunek, J., K.L. Bristow, S.A. Helalia, and A.A. Siyal. (2016). The effect of different fertigation strategies and furrow surface treatments on plant water and nitrogen use. Irrig Sci., 34: 53-69.

Tilly, N., D. Hoffmeister, Q. Cao, V. LenzWiedemann, Y. Miao, and G. Bareth. (2015). Transferability of models for estimating paddy rice biomass from spatial plant height data. Agriculture, 5, 538-560. DOI: https:// doi.org/10.3390/agriculture5030538

Wang, J., X. Lv, W. Jiang-li, and L. Hai-rong. (2014). Spatiotemporal Variations of Reference Crop Evapotranspiration in Northern Xinjiang, China. The Scientific World Journal, 10 pages. DOI: http://dx.doi.org/ 10.1155/ 2014/931515. 